\title{
ON GRAPH FOLDING AND MOBILE RADIO FREQUENCY ASSIGNMENT
}

\author{
F. Salama* and H. Rafat** \\ Mathematics Department, Faculty of Science, Tanta University, Tanta, Egypt \\ *Email: fatma.salama@science.tanta.edu.eg \\ **E-mail: hesham.abdelmaksoud@science.tanta.edu.eg
}

Received 13/2/2018

Revised 3/3/2018

Accepted 13/4/2018

\begin{abstract}
In this paper, the definition of a jumping folding of a graph has been introduced. The relations between this type of jumping folding of the graph and its chromatic number will be obtained. Theorems governing these relations have been introduced. Mobile radio frequency assignment has been given as an application.

Keywords: Folding, Graph, Vertex-coloring, Chromatic Number.

\section{Mathematics subject classification: $05 \mathrm{C} 15,05 \mathrm{C} 99,05 \mathrm{C} 90,05 \mathrm{C} 30$ 1. Introduction:}

Coloring is the most important area of study in graph theory. This topic has many applications in our life. Let $G$ be a graph and $m$ be a positive integer, a $m$-coloring is a function $M: V(G) \rightarrow\{1, \ldots, m\}$ from the set of vertices into the positive integers set less than or equal to $m$. We say that $M$ is a proper $m$-coloring of $G$ if for every pair $u, v$ of adjacent vertices, $M(u)=M(v)$ that is, if adjacent vertices are colored differently. If such a coloring exists for a graph $G$, we say that $G$ is $m$-colorable. Given a graph $G$, the chromatic number of $G$, denoted by $\chi(G)$, is the minimum value of $m$ such that $G$ admits a $m$-coloring. That is, $\chi(G)$ is the smallest number of colors needed to color the vertices of $G$ in such a way that adjacent vertices are colored by different colors (for more details see [1-11]).

In [12], S. A. Robertson introduced the definition of folding on manifolds. P. Di. Francesco in [13] reviewed various folding problems with respect to the related coloring problems. J. Nešetril a , P. O. de Mendez in [14] defined folding of a directed graph as a coloring. While in general folding is as complicated as homeomorphisms for some classes they present useful tool to study colorings and homeomorphisms.
\end{abstract}


The above discussion motivates us to define new type of graph folding that helps to calculate the chromatic number of the graph by easy way.

\section{The main results:}

We introduce the following definitions:

Definition 1. In any graph $G$ a vertex $v_{i}$ is an n-pure neighbor of the vertex $v_{j}$ if the shortest length of path between them is $n, n \geq 1$.

Definition 2. A jumping folding $F$ of a graph $G$ is a map from the graph $G$ to a graph $G^{*}, F: G \rightarrow G^{*}$, folded only one vertex $v_{i}$ to one of its 2- pure neighbor and any other one to itself.

To illustrate this definition, consider we have a graph $G$ in Fig. (1),

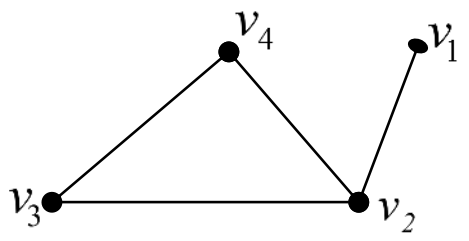

Fig. (1)

The vertex $v_{1}$ has two vertices $v_{3}$ and $v_{4}$ are $2-$ pure neighbor of it. So, we can find jumping folding $F$ from the graph $G$ to a graph $G^{*}$, i.e. $F: G \rightarrow G^{*}$, such that $F\left(v_{1}\right)=v_{3}, F\left(v_{i}\right)=v_{i}, i \in\{2,3,4\}$. We notice that $G^{*}=$ $F(G)$ is a subgraph of $G$, see Fig. (2).
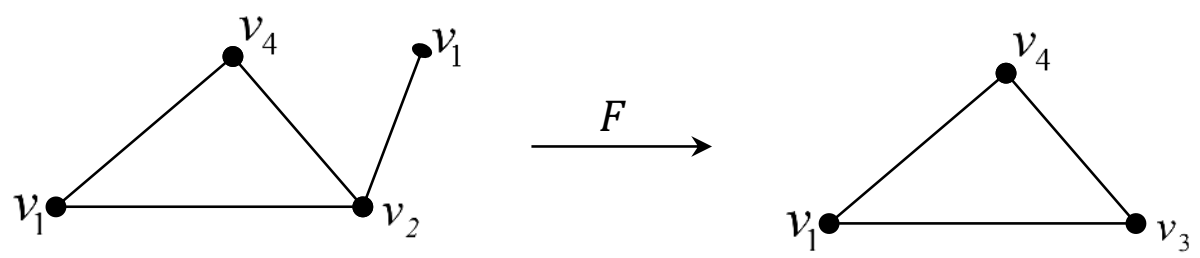

Fig. (2)

In the following discussion we will find the relation between the end of jumping folding of graph and its chromatic number.

Let we have a graph $G_{1}$, as shown in Fig. (3), the chromatic number of this graph equals 3, i.e. $\chi\left(G_{1}\right)=3$.

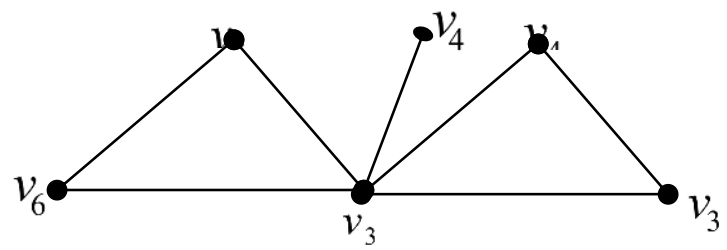

Fig. (3) 
Also, let we have jumping folding $F_{1}: G_{1} \rightarrow G_{2}$, such that $F_{1}\left(v_{1}\right)=v_{3}, F_{1}\left(v_{i}\right)=v_{i}, \mathrm{i} \in\{2,3,4,5,6\}$.

As shown in Fig. (4) the graph after folding $G_{2}=F_{1}\left(G_{1}\right)$, its chromatic number $\chi\left(G_{2}\right)=3$ and its order equals 5.

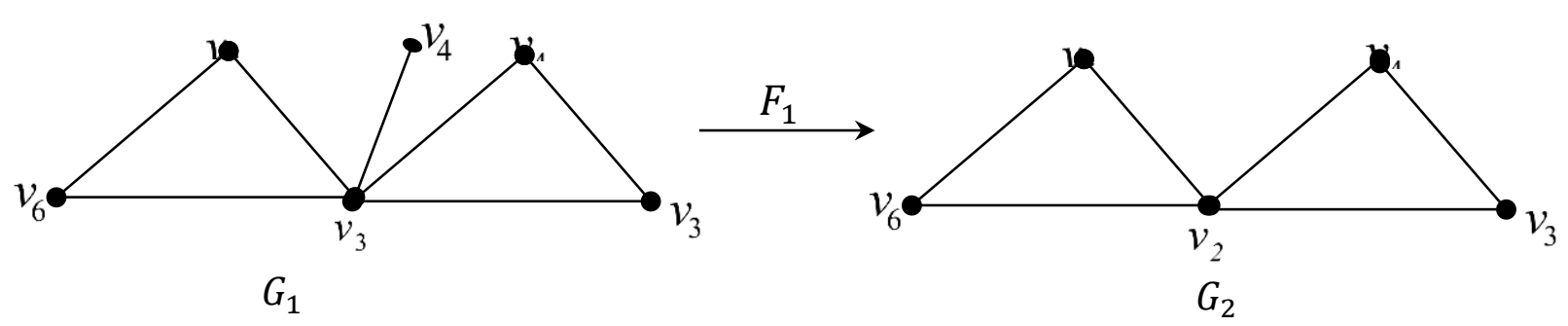

Fig. (4)

Since the graph $G_{2}$ has the vertex $v_{4}$ which is $2-$ neighbor of the vertex $v_{5}$. Then we can find another jumping folding $F_{2}: G_{2} \rightarrow G_{3}$, such that $F_{2}\left(v_{4}\right)=v_{5}, F_{2}\left(v_{i}\right)=v_{i}, \mathrm{i} \in\{2,3,5,6\}$. In this case the graph after folding $G_{3}=$ $F_{2}\left(G_{2}\right)$ has chromatic number $\chi\left(G_{3}\right)=3$ and its order equals 4 , see Fig. (5).

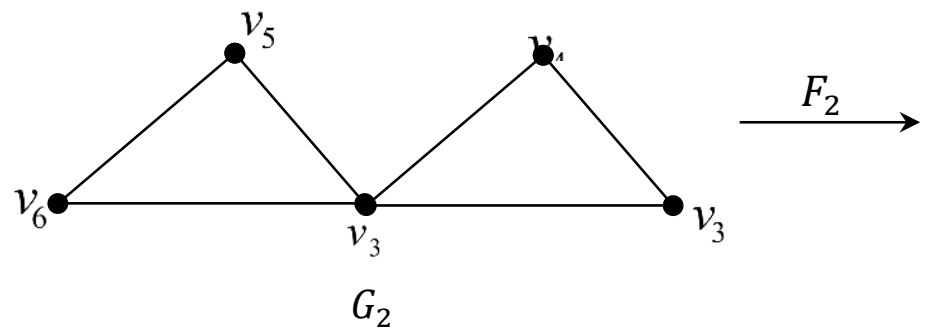

Fig. (5)

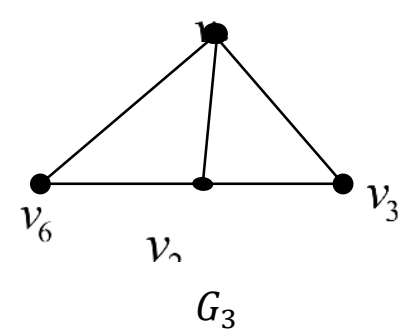

Since the graph $G_{3}$ has the vertex $v_{3}$ which is 2 -pure neighbor of the vertex $v_{6}$. Then the jumping folding $F_{3}: G_{3} \rightarrow G_{4}$ can be found, such that $F_{3}\left(v_{3}\right)=v_{6}, F_{3}\left(v_{i}\right)=v_{i}, \mathrm{i} \in\{2,5,6\}$. In this case, the chromatic number of $G_{4}=F_{3}\left(G_{3}\right)$ is $\chi\left(G_{4}\right)=3$ and its order equals 3, see Fig.(6).

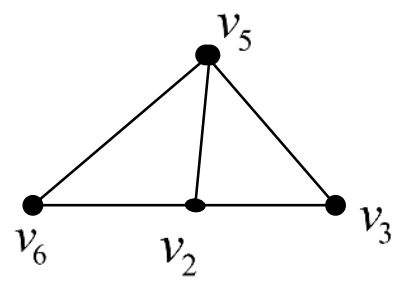

$G_{3}$

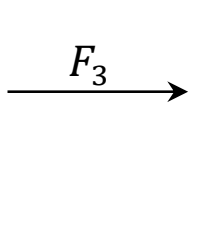

Fig. (6)

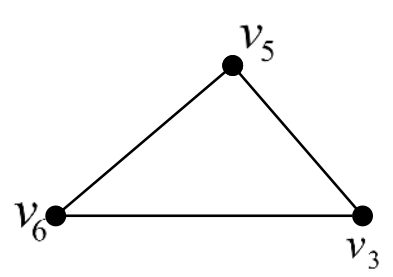

$G_{4}$ 
From the above discussion we notice that:

(1) The graph $G_{4}$ does not have any vertex which is 2 -pure neighbor to another, i.e. $G_{3}$ is complete graph and $F_{3}$ is the end of jumping folding of $\mathrm{G}_{1}$.

(2) The vertices $v_{3}, v_{1}$ and $v_{6}$ can be colored by the same color and also the vertices $v_{4}, v_{5}$ have the same color but $v_{2}$ should have different color.

(3) $\chi\left(G_{4}\right)=\chi\left(G_{1}\right)$.

(4) The chromatic number of $G_{4}$ equals its order.

Proposition 1. The end of a jumping folding of a connected graph is a complete graph.

Proof. Let a graph $G^{*}$ be the end of jumping folding of a connected graph $\mathrm{G}$ and let $G^{*}$ be not a complete graph. Since $G^{*}$ is the end of jumping folding of $\mathrm{G}$ then $G^{*}$ does not have any vertex that 2 -pure neighbor to another one, i.e. every vertex in $\mathrm{G}^{*}$ is connected to the others. That is a contradiction and hence $\mathrm{G}^{*}$ is a complete graph.

Proposition 2. In any graph if a vertex is folded by jumping folding to another one then these two vertices have the same color.

Theorem 1. For any connected graph that cannot be folded by jumping folding, the chromatic number of this graph is equal to its order.

Proof. Let we have a connected graph $G$ which cannot be folded by jumping folding. This graph does not have any vertex which is $2-$ neighbor to another one, i.e. every vertex is connected to the others. Hence, this graph is a complete graph and its chromatic number equals its order.

Theorem 2. If any disconnected graph cannot be folded by jumping folding then the chromatic number of this graph is $\chi(\mathrm{G})=\left\{\begin{array}{ll}1 & \text { if } m=0 \\ \frac{1+\sqrt{1+8 m}}{2} & \text { if } m \neq 0\end{array}\right.$, where $m$ is the size of the graph.

Proof. Let we have a disconnected graph $G$ which cannot be folded by jumping folding. Then this graph does not have any vertex which is 2 -neighbor to another one. Then this graph is one of these cases:

Case 1: the graph is null graph. Hence its chromatic number equals one.

Case 2: the graph is the union of two graphs one is null and the other is complete. Then the chromatic number of our graph equals to the chromatic number of the complete one which its chromatic number equals it's order $=\frac{1+\sqrt{1+8 m}}{2}$ where $m$ is the size. Hence the chromatic number of our graph $\chi(\mathrm{G})=\frac{1+\sqrt{1+8 m}}{2}$. 


\section{Application:}

Frequency Assignment of Mobile Radio: It is known that, frequencies assigned to all towers must be different at the same location to minimize the interference level between towers. The plan in Fig.(7) shows many levels of interference and we want to calculate the minimum number of frequencies needed.

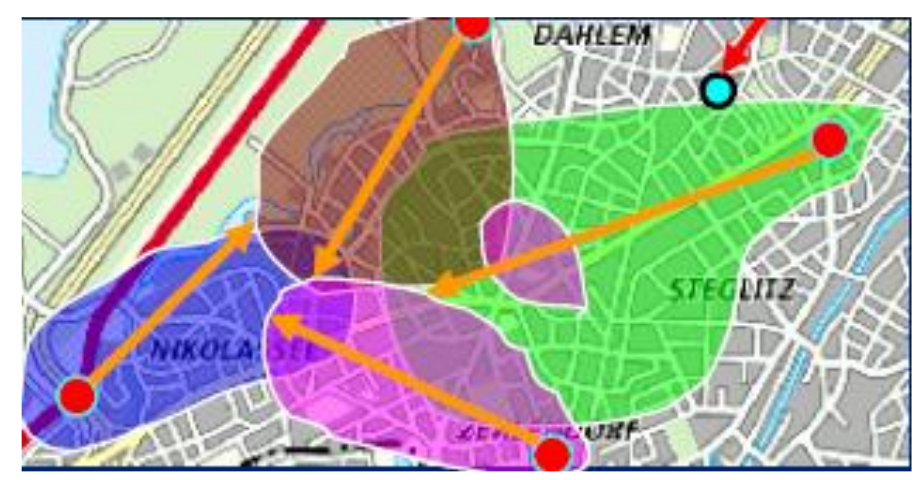

Fig. (7)

To solve this problem, we model the plan as a graph such that the vertices represent towers and edges represent separation constraints, see Fig. (8).

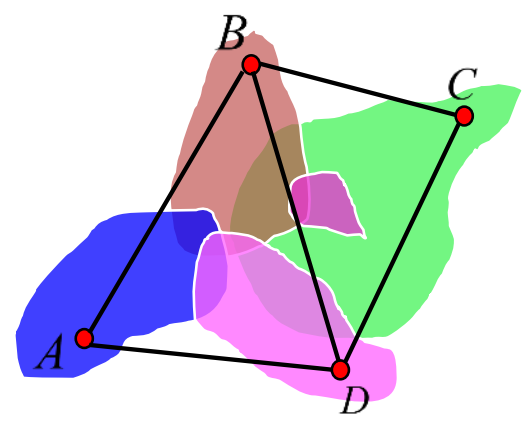

Fig. (8)

The vertex $A$ in the graph shown in Fig.( 8 ) is a 2 -pure neighbor of $C$. So we can find a jumping folding $f$ where $f(C)=A$ and $f(I)=I, \forall I \in\{A, B, D\}$.

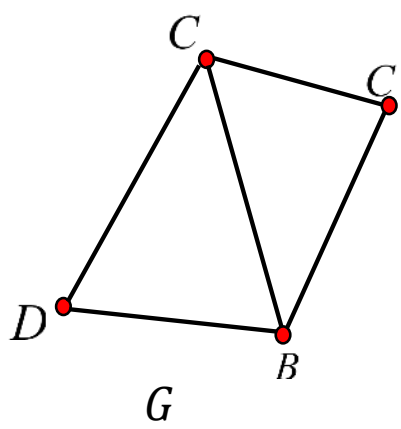

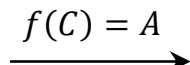

Fig. (9)

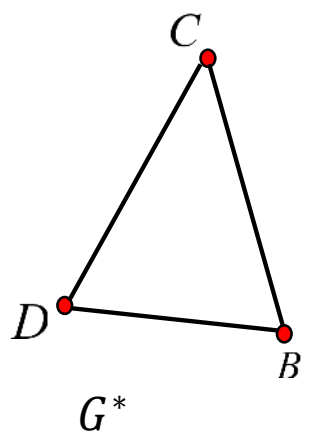

$G^{*}$ 
Since the graph $G^{*}$ is a complete graph, then $\chi(\mathbf{G})=\chi\left(\mathbf{G}^{*}\right)=\mathbf{3}$. Hence the minimum number of frequencies needed is 3 .

\section{Conclusion}

A new type of graph folding has been defined. The relation between this type of graph folding and its chromatic number has been found. Theorems governing this relation have been proven. We have taken the frequency assignment of mobile radio as an application.

\section{Acknowledgment}

We are so grateful to the reviewer for his many valuable suggestions and comments that significantly improved the paper.

\section{References}

[1] J.A. Bondy and U.S.R. Murty: Graph Theory, Graduate Texts in Mathematics series,Springer,2008.

[2] O.V. Borodin: Colorings of plane graphs, Discrete Mathematics, Elsevier,313, 315-539, 2013.

[3] C. J. Casselgren ,H. H. Khachatrian and P. A. Petrosyan: Some bounds on the number of colors in interval and cyclic interval edge colorings of graphs, Discrete Mathematics 341, 627-637, 2018.

[4] K. R. Chandrasekar: Edge irredundant colorings in graphs, Electronic Notes in Discrete Mathematics 63, 211-218, 2017.

[5] M. Demange, T. Ekim, B. Ries and C. Tanasescu : On some applications of the selective graph coloring problem, European Journal of Operational Research, Elsevier ,240, 307-314, 2015.

[6] M. El-Ghoul, F. Salama and A. S. Pelila: Folding of a 2-Tangle-Graphs, Journal of Mathematics Research, Canadian Center of Science and Education, 4, 86-100, 2012.

[7] M. C. Golumbic: Total coloring of rooted path graphs, Information Processing Letters 135, 73-76, 2018.

[8] S.J. Kim and B. Park: List 3-dynamic coloring of graphs with small maximum average degree, Discrete Mathematics 341,1406-1418, 2018.

[9] M. Molloy and B. Reed: Colouring graphs when the number of colours is almost the maximum degree, Journal of Combinatorial Theory, Series B, Elsevier, 109, 134-195, 2014.

[10] I. Tomon: On the chromatic number of regular graphs of matrix algebras, Linear Algebra and its Applications, Elsevier, 475, 154-162, 2015.

[11] V. I. Voloshin: Introduction to graph theory. Nova Science Publishers, Company, Inc, New York, 2009.

[12] S. A. Robertson: Isometric folding of Riemannian manifolds, Proceeding of the Royal Society of Edinburgh, 79, 275-284, 1977.

[13] P. Di-Francesco: Folding and coloring problem in mathematics and physics, Bulletin of the American Mathematics Society, 37, 251-307, 2000.

[14] J. Nešetril a, P. O. de Mendez: Folding, Journal of Combinatorial Theory, Series B, Elsevier, 96, no.5, 730$739,2006$. 